MATHEMATICAL ASSOCIATION

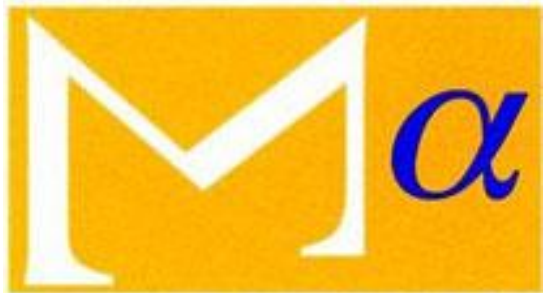

supporting mathematics in education

Note on the Addition Formulae of Trigonometry

Author(s): J. G. Leathem

Source: The Mathematical Gazette, Vol. 3, No. 47 (Oct., 1904), pp. 81-85

Published by: Mathematical Association

Stable URL: http://www.jstor.org/stable/3603633

Accessed: 26-11-2015 05:43 UTC

Your use of the JSTOR archive indicates your acceptance of the Terms \& Conditions of Use, available at http://www.jstor.org/page/ info/about/policies/terms.jsp

JSTOR is a not-for-profit service that helps scholars, researchers, and students discover, use, and build upon a wide range of content in a trusted digital archive. We use information technology and tools to increase productivity and facilitate new forms of scholarship. For more information about JSTOR, please contact support@jstor.org. 
making $O Y$ equal to $O Z$. Join $A Y$, and produce it to $E$, making $A E$ equal to $A C$. Join $C E$; then $C E$ is the required perpendicular.

One perpendicular can always be drawn from $C$ to $A B$, and not more than one, unless every line through $C$ is perpendicular to $A B$.

(5) To bisect a given segment. (Fig. 5.)

Let $A B$ be the segment. Draw $A C, B D$ perpendicular to $A B$ on opposite sides, and make $A C$ equal to $B D$. Then $C D$ cuts the segment $A B$ at its middle point $O$.

If straight lines are closed, $C$ must not be a point such that every line through $C$ is perpendicular to the line $A B$, and $C$ must not be at the second

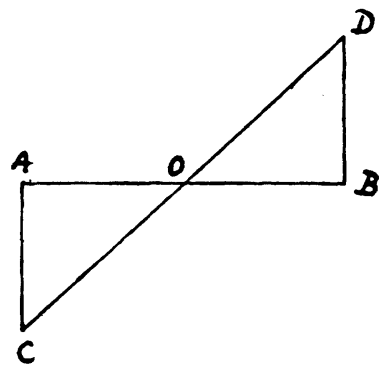

Fig. 5.

point of intersection of the lines $A C, A B$ if there is one. The proof is by first showing that $O C=O D$; and then showing that if $O A$ is not equal to $O B$ a perpendicular can be drawn from $C$ to $A B$ different from $C A$, which is impossible. An open segment $A B$ has always two opposite sides, although a closed straight line may not have two sides.

(6) To draw a line making with a given line an angle equal to a given angle. (Fig. 6.)
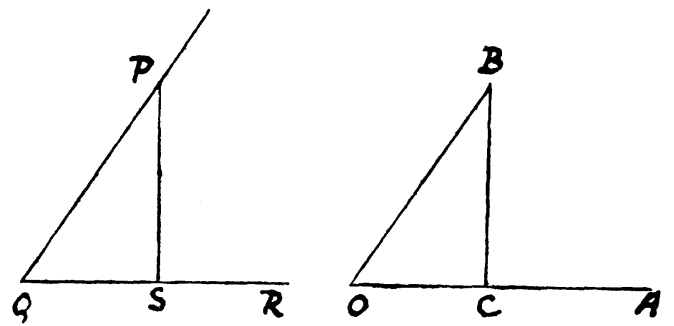

Fig. 6.

Let $O A$ be the given line, and $P Q R$ the given angle. From a point $P$ on $Q P$ draw $P S$ perpendicular to $Q R$. From $O A$ cut off $O C$ equal to $Q S$. Draw a perpendicular $C B$ to $O A$, and make $C B$ equal to $S P$. Join $O B$; then $A O B$ is the required angle.

F. S. Macaulay.

\section{NOTE ON THE ADDITION FORMULAE OF TRIGONOMETRY.}

Proofs of the addition formulae for angles of any magnitudes are sometimes rendered invalid by the way in which arguments are founded on a diagram which can represent only one of the many possible cases. When the two 
angles under consideration are represented in the usual manner as having a common vertex, the only angles in the diagram whose values it is safe to represent by formulae, if complete generality is to be preserved, are angles having this same vertex. The introduction of other angles may be avoided by a restatement of the definitions of sine and cosine.

At the outset some remarks about the specification of an angle suggest themselves.

(1) When we speak of the angle between two lines we mean the angle between two directions; it is therefore desirable, even in the most elementary teaching, to postulate that the trigonometrical line, that is a line regarded as an arm of an angle, shall always mean a directed line, a line with an arrowhead.

(2) The merely arithmetic idea of an angle is sufficient in elementary Trigonometry, and it is unwise to suggest to the beginner that an angle described in the clockwise sense must be regarded as negative ; the tablebook tells us the value of the sine of an angle without asking in which sense the angle is supposed to be described, and the learner ought to be led to take the same point of view. In elementry Trigonometry and in practical work the measure of an angle is an arithmetical quantity, subject to arithmetic addition and subtraction; the idea of algebraic addition, with the implied idea of a negative angle, is confusing to the beginner and may with advantage be postponed.

(3) Two directed lines are the arms of an infinite series of angles. When we speak of the angle between two lines we mean one of these infinitely numerous angles, and we must know which. The manner of obtaining this knowledge does not concern us at present, but it may be remarked here that in the most frequent application, namely to the triangle, our choice is conventionally directed to the interior angles. The essential thing is that we know what angle is to be discussed before we discuss it; the method of indicating an angle in a diagram by means of an arc of a small circle is, of course, most valuable.

\section{Definitions.}

Either arm of an angle may be called the first arm, and the angle may be regarded as described by a line starting from the first arm and revolving through the angle to the second arm. The sense in which the revolution takes place depends on which arm we select as the first arm. In order to define the sine and the cosine a choice must be made of the order in which the sides are to be taken, but the choice is quite arbitrary.

Let $O A, O B$ be the arms of an angle $\theta$, and let $O A$ be chosen as the first arm. Of the two directed lines through $O$ at right angles to $O A$ let $O R$ be that which is such that the right angle $A O R$ and the angle $\theta$ would be described in the same sense by a revolving line starting from $O A$; the directed line $O R$ may be called the right-angle arm corresponding to the angle $\theta$. But it will perhaps be more convenient to call $O A$ the cosine arm, $O B$ the second $a r m$, and $O R$ the sine arm of the angle $\theta$. Of course if $O B$ were taken as cosine arm we should get a different sine arm.

Now measure a unit distance $O P$ from $O$ along the second arm $O B$ of the angle, in the direction of the arrow belonging to $O B$. The cosine of $\theta$ is defined to be the projection of $O P$ on the cosine arm $O A$; the sine of $\theta$ is defined to be the projection of $O P$ on the sine arm $O R$; projections are to be estimated algebraically, with reference to the arrows on $O A$ and $O R$.

These definitions have the advantage that they make no mention of 'right,' 'left,' 'up,' 'down,' 'clockwise' or 'counterclockwise.' It follows from them that if $O Q$ be any length, positive or negative, measured along the second arm of an angle $\theta$, its projections on the cosine arm and sine arm respectively are algebraically equal to $O Q \cos \theta$ and $O Q \sin \theta$. 
To find the sine and cosine of $\frac{1}{2} \pi+\theta$.

Let $A O B$ be the angle $\theta$, and let $O A$ be taken as cosine arm. Let $O R$ be the corresponding sine arm, and let $R O$ be produced to $S$. Then the angle

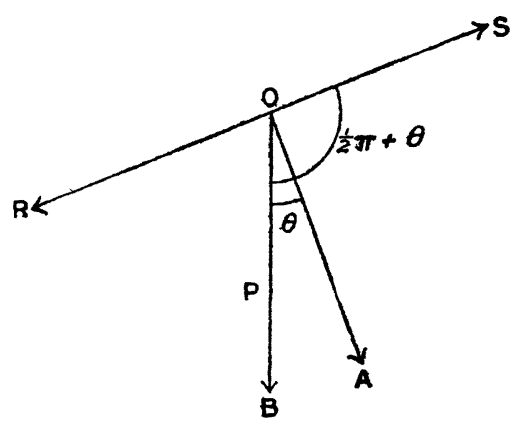

$S O B$ is $\frac{1}{2} \pi+\theta$, and we may take $O S$ for its cosine arm. Thus $\theta$ and $\frac{1}{2} \pi+\theta$ have the same second arm $O B$. Let $P$. be in $O B$ at unit distance from $O$.

Then $O A$ is the sine arm of $\frac{1}{2} \pi+\theta$, and is the cosine arm of $\theta$. Hence $\cos \theta$ and $\sin \left(\frac{1}{2} \pi+\theta\right)$ are both equal to the projection of $O P$ on $O A$, and so are equal to one another.

Again $O R$ is the sine arm of $\theta$, and $O S$ is the cosine arm of $\frac{1}{2} \pi+\theta$, and these are in opposite directions. The projection of $O P$ on $O S$ is minus the projection of $O P$ on $O R$ : hence $\cos \left(\frac{1}{2} \pi+\theta\right)=-\sin \theta$.

\section{The Addition Formulae.}

Let a straight line start from coincidence with $O A$ and revolve about $O$ always in the same sense. Let $O B$ be its position when it has described an

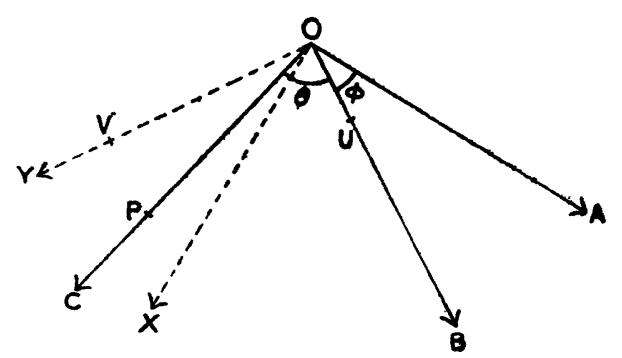

angle $\phi, O C$ its position when it has described $\phi+\theta, O X$ its position when it has described $\frac{1}{2} \pi$, and $O Y$ its position when it has described $\frac{1}{2} \pi+\phi$.

Then we may take $O A, O B, O X$ for cosine arm, second arm, and sine arm respectively of the angle $\phi$ or $A O B$; we may take $O B, O C, O Y$ for cosine arm, second arm, and sine arm respectively of the angle $\theta$ or $B O C$; and we may take $O A, O C, O X$ for cosine arm, second arm, and sine arm respectively of the angle $\phi+\theta$ or $A O C$.

Let $P$ be at unit distance from $O$ on $O C$, and let $O U, O V$ be the projections of $O P$ on $O B$ and $O Y$; of course $U P$ and $O V$ are parallel and in the same direction.

The cosine of $\phi+\theta$ is the projection of $O P$ on $O \overline{O F}$, this is the same as the sum of the projections of $O U$ and $U P$ (or $O V$ ) on $O A$. But $O U$ is $\cos \theta$, and 
is estimated positively along the second arm $O B$ of the angle $\phi$ whose cosine arm is $O A$; so the projection of $O U$ on $O A$ is $\cos \theta \cos \phi$. And $O V$ is $\sin \theta$, and is estimated positively along the second arm $O Y$ of the angle $\frac{1}{2} \pi+\phi$ whose cosine arm is $O A$; so the projection of $O V$ on $O A$ is $\sin \theta \cos \left(\frac{1}{2} \pi+\phi\right)$.

Hence

$$
\begin{aligned}
\cos (\phi+\theta) & =\cos \theta \cos \phi+\sin \theta \cos \left(\frac{1}{2} \pi+\phi\right) \\
& =\cos \theta \cos \phi-\sin \theta \sin \phi .
\end{aligned}
$$

The sine of $\phi+\theta$ is the sum of the projections of $O U$ and $O V$ on $O X$. Now $O X$ is the sine arm of the angle $\phi$ along whose second arm lies $O U$, and also of the angle $\frac{1}{2} \pi+\phi$ along whose second arm lies $O V$. Hence

$$
\begin{aligned}
\sin (\phi+\theta) & =O U \sin \phi+O V \sin \left(\frac{1}{2} \pi+\phi\right) \\
& =\cos \theta \sin \phi+\sin \theta \cos \phi .
\end{aligned}
$$

\section{The Subtraction Formulae.}

Let a straight line start from coincidence with $O A$ and revolve about $O$, always in the same sense. Let $O B$ be its position when it has described an

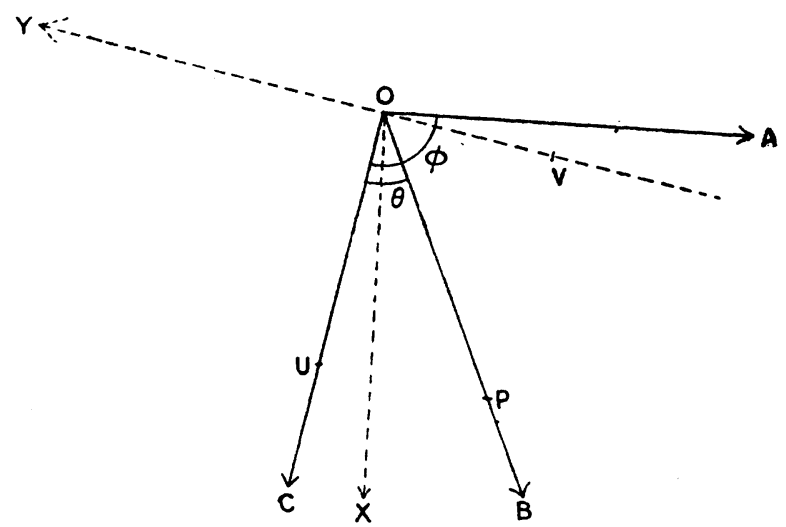

angle $\phi-\theta, O C$ its position when it has described an angle $\phi, O X$ its position when it has described $\frac{1}{2} \pi$, and $O Y$ its position when it has described $\frac{1}{2} \pi+\phi$.

Then we may take $O A, O B, O X$ for cosine arm, second arm, and sine arm of the angle $\phi-\theta$ or $A O B$; we may take $O A, O C, O X$ for cosine arm, second arm, and sine arm of the angle $\phi$ or $A O C$; and we may take $O C, O B$, and the line $O Y$ reversed as cosine arm, second arm, and sine arm of the angle $\theta$ or $B O C$.

Let $P$ be at unit distance from $O$ along $O P$, and let $O U, O V$ be the projections of $O P$ on $O C$ and $O Y$ respectively. Then $O U$ is $\cos \theta$; but $O V$, being estimated positively along $O Y$ which is the reversed sine arm of $\theta$, is $-\sin \theta$.

The cosine of $\phi-\theta$ is the projection of $O P$ on $O A$; this is the same as the sum of the projections of $O U$ and $U P$ (or $O V$ ) on $O A$. But $O U$ is measured along $O C$, the second arm of the angle $\phi$ whose cosine arm is $O A$; so the projection of $O U$ on $O A$ is $O U \cos \phi$, or $\cos \theta \cos \phi$. And $O V$ is measured along $O Y$, the second arm of the angle $\frac{1}{2} \pi+\phi$ whose cosine arm is $O A$; so the projection of $O V$ on $O A$ is $O V \cos \left(\frac{1}{2} \pi+\phi\right)$ or $-\sin \theta \cos \left(\frac{1}{2} \pi+\phi\right)$. Hence

$$
\begin{aligned}
\cos (\phi-\theta) & =\cos \theta \cos \phi-\sin \theta \cos \left(\frac{1}{2} \pi+\phi\right) \\
& =\cos \theta \cos \phi+\sin \theta \sin \phi .
\end{aligned}
$$


The sine of $\phi-\theta$ is the sum of the projections of $O U$ and $O V$ on $O X$. Now $O X$ is the sine arm of the angle $\phi$ along whose second arm lies $O U$, and also of the angle $\frac{1}{2} \pi+\phi$ along whose second arm lies $O V$. Hence

$$
\begin{aligned}
\sin (\phi-\theta) & =O U \sin \phi+O V \sin \left(\frac{1}{2} \pi+\phi\right) \\
& =\cos \theta \sin \phi-\sin \theta \sin \left(\frac{1}{2} \pi+\phi\right) \\
& =\cos \theta \sin \phi-\sin \theta \cos \phi .
\end{aligned}
$$

\section{Negative Angles.}

The transition from arithmetic subtraction to algebraic addition is effected by giving to the sine and cosine of a negative angle such meanings as shall make the subtraction formulae particular cases of the addition formulae. It is easily seen that the required convention is simply that when an angle is to be regarded as negative the direction of its sine arm must be reversed.

St. John's College, Cambridge.

J. G. Leathem.

\section{A SUGGESTED REARRANGEMENT OF THE BOOK- WORK ON SOME ELEMENTARY SERIES.}

IN all the English text-books on Analytical Trigonometry, so far as I know, the power-series for $\cos x$ is obtained as the limit, when $n$ tends towards* infinity, of the finite sum for $\cos x$,

$$
\cos ^{n}\left(\frac{x}{n}\right)\left[1-\left(1-\frac{1}{n}\right) \frac{x^{2}}{2 !} t^{2}+\left(1-\frac{1}{n}\right)\left(1-\frac{2}{n}\right)\left(1-\frac{3}{n}\right) \frac{x^{4}}{4 !} t^{4}-\ldots\right]
$$

where $t=\left(\tan \frac{x}{n}\right) / \frac{x}{n}$ and the number of terms is equal to the integer next greater than $\frac{1}{2} n$. The power-series for $\sin x$ is obtained similarly; the two series and the method of finding them being both due to Euler.

Now it appears to me that this method is subject to certain drawbacks, when introduced in a first course on Analytical Trigonometry. For there are two ways of calculating the limit; the harder (but accurate) one as given in books such as Chrystal's Algebra or Hobson's Trigonometry; and the easier (inaccurate) method of the older text-books, which has unfortunately been reproduced in some new texts. +

The weakness in the inaccurate form of the proof is found in the assumption that the product

$$
\left(1-\frac{1}{n}\right)\left(1-\frac{2}{n}\right) \ldots\left(1-\frac{2 r-1}{n}\right)
$$

has the limiting value 1 , for all values of $r$ which do not exceed $\frac{1}{2} n$ (see for instance pp. 109, 110 of Lachlan and Fletcher's book just quoted). The falsity of this assumption is almost self-evident, when thus stated; but the assumption is, nevertheless, nearly always swallowed by the beginner without a qualm. $\neq$

\footnotetext{
* It is perhaps worth while to enter a plea for the use of the phrase, from the very beginning, in dealing with limits. The phrases "the limit when $n$ is $\infty$," "the limit when $n=\infty$ " can do nothing but harm.

+ Castle's Manual of Practical Mathematics (Macmillan, 1903) ; Lachlan and Fletcher's Elements of Trigonometry (Arnold, 1904). I refer to these as examples, merely ; in many other respects both books strike me as good.

$\mp$ I have found a little numerical calculation very useful in convincing the unbeliever that there $i s$ an assumption involved. Thus with $n$ even, and $v=\frac{1}{2} n$, we find the product to be less than $1 / 10^{3}$ when $n=10$; less than $1 / 10^{10}$ for $n=30$; less $\operatorname{than} 1 / 10^{20}$ for $n=50$.
} 